\title{
Jovens e metas para o futuro: Uma revisão crítica da literatura
}

\author{
Dandara de Oliveira Ramos \\ Maria Lucia Seidl-de-Moura \\ Universidade do Estado do Rio de Janeiro \\ Luciana Fontes Pessôa \\ Pontifícia Universidade Católica do Rio de Janeiro
}

\begin{abstract}
Resumo
O presente trabalho apresenta uma revisão da literatura sobre de metas de realização e objetivos futuros de jovens, visando identificar as principais orientações teóricas que norteiam os estudos feitos e discutilas sob a ótica da Psicologia Evolucionista. Três eixos teóricos principais foram encontrados na revisão realizada: (a) estudos baseados na Teoria da Perspectiva do Tempo Futuro; (b) estudos baseados na Teoria da Autodeterminação; e (c) estudos dedicados à investigação de fatores neurais e cognitivos associados ao desenvolvimento de metas para o futuro. Com base nas teorias do Desconto do Futuro e das Estratégias de História de vida, propostas pela Psicologia Evolucionista, sintetizamos um modelo diferenciado para o estudo do tema, envolvendo variáveis sociodemográficas, econômicas e contextuais específicas.
\end{abstract}

Palavras-chave: metas futuras; jovens; perspectiva de futuro; revisão da literatura; psicologia evolucionista.

\begin{abstract}
Youth and goals for the future: A critical bibliographic review. This article presents a literature review about future goals of young people, aiming to identify the main theoretical orientations in which are based the studies conducted and to discuss them under the Evolutionary Psychology perspective. Three central theoretical orientations were identified: (a) studies based on the Future Time Perspective Theory, (b) studies based on the Self Determination Theory, and (c) other studies dedicated to the cognitive and neural processes associated with the development of goals for the future. Based on Future Discounting and Life History Theory proposed by Evolutionary Psychology, we propose a different framework for the study of this topic, including socio-demographic, economics and specific contextual variables.
\end{abstract}

Keywords: future goals; youth; future perspective; literature review; evolutionary psychology.

\section{Resumen}

Jóvenes y metas para el futuro: Una revisión crítica de la literatura. En este trabajo se presenta una revisión de la literatura sobre las metas de logro y las metas futuras de los jóvenes, para identificar las principales orientaciones teóricas que guían los estudios y las discutimos desde la perspectiva de la Psicología Evolutiva. Tres grandes ejes teóricos fueron encontradas en la revisión llevada a cabo: (a) estudios basados en la Teoría de la Perspectiva del Tiempo Futuro, (b) estudios basados en la Teoría de Autodeterminación, y (c) estudios dedicados a la investigación de los factores neurales y cognitivos asociados con el desarrollo de metas para el futuro. Con base en las teorías de Descuento Futuro y Estrategias de Historia de Vida, propuestas por la Psicología Evolutiva, sintetizamos un modelo diferenciado para el estudio del tema, con participación de variables sociodemográficas, económicas y contextuales especificas.

Palabras clave: metas futuras; juventud; perspectivas de futuro; revisión de la literatura; psicología evolutiva.

$\mathrm{A}$ juventude, e em especial a adolescência, são consideradas como os períodos mais importantes do desenvolvimento humano no que tange à construção de metas e objetivos futuros para um projeto de vida (Damon, 2003). Autores dedicados ao estudo das teorias da identidade e do desenvolvimento apontam a juventude como o estágio do ciclo vital no qual as pessoas começam a manifestar claramente os sistemas de crenças que fundamentarão as ações voltadas ao meio externo relativas aos projetos que desejam realizar no futuro (Baumeister, 1995; Erikson, 1968; Loevinger, 1976; Marcia, 2002; Montemayor, 1983). Este processo, no entanto, não é uniforme. Alguns jovens em situações específicas podem não manifestar seus interesses e 
engajamento em projetos futuros com tal clareza, ou até mesmo podem apresentar dificuldades em declarar expectativas quanto à própria noção de futuro (Ramos, 2009).

Cabe, no entanto, nos perguntarmos qual é o espaço que vem sendo dado ao jovem na sociedade contemporânea, e também nos questionarmos acerca do modo como entendemos e teorizarmos seu desenvolvimento. O comportamento dos jovens com relação ao futuro, de acordo com a maioria das teorias, é concebido como uma combinação dos seguintes fatores: predisposições genéticas, gênero, influência do ambiente social e histórico, condições econômicas, práticas culturais, experiências iniciais de cuidados parental recebidos, ordem de nascimento, relação com outros sujeitos jovens e com os familiares, e a escolarização (Baron, 2008). As teorias diferem, no entanto, na forma de abordar e relacionar tais aspectos, assim como privilegiam, ou por vezes renegam, uns em detrimento de outros.

O presente trabalho apresenta uma revisão da literatura a respeito do processo de construção de metas de realização e objetivos futuros de jovens. O objetivo central do trabalho é apresentar os principais eixos teóricos que embasam o estudo do tema e promover uma reflexão com base na proposta da Psicologia Evolucionista.

\section{Método}

Para identificar, localizar e adquirir as publicações de interesse foram consultadas as bases de dados ISI Web of Knowledge (http://isiknowledge.com) e o portal de Periódicos da Coordenação de Aperfeiçoamento de Pessoal de Nível SuperiorCAPES (http://periodicos.capes.gov.br). A utilização do portal da CAPES é justificada devido a sua abrangência e facilidade de acesso para a maioria dos pesquisadores no Brasil. Na consulta dos periódicos buscou-se como palavras-chave, os termos metas para o futuro, metas de realização, jovens e futuro, perspectiva de futuro e projetos de vida (utilizaram-se também os termos em inglês: future goals, achievement goals, future achievement, future perspective, youth and future, life project). Realizou-se a procura nos abstracts e, quando esta primeira opção não estava disponível na base consultada, foi feita uma investigação no texto completo.

Também foi efetuada um levantamento nas bases de dados SciELO Brasil, PsycNet, BVS Psi e Scholar Google, utilizando as mesmas palavras-chave. O recorte temporal do estudo considerou artigos publicados nos últimos dez anos (2002-2012), dos quais foram adquiridos os textos de acesso livre.

\section{Resultados}

A busca realizada gerou a identificação de um total de 89 artigos. A partir dos resumos, alguns destes artigos foram considerados mais relevantes para a composição da discussão de cada um dos eixos e foram selecionados 26 artigos para aprofundamento. Além disso, eventualmente outras referências bibliográficas indicadas nos artigos também foram consultadas, sendo inseridas no corpo desse trabalho. Três eixos teóricos principais foram encontrados na revisão realizada: (a) estudos baseados na Teoria da Perspectiva do Tempo Futuro; (b) estudos baseados na Teoria da Autodeterminação; e (c) estudos dedicados à investigação de fatores neurais e cognitivos associados ao desenvolvimento de metas para o futuro.

Avaliando o material disponível sobre metas de realização de jovens, pode-se afirmar que algumas teorias predominam nesse campo de estudo e que não há muita diversidade nos trabalhos e pesquisas sobre este tema. Nos últimos anos, os estudiosos vêm se limitando a replicar experimentos clássicos, comparando seus resultados entre situações específicas. As seções seguintes apresentam uma apreciação crítico-reflexiva do material consultado, conforme os principais eixos teóricos encontrados.

\section{Teoria da Perspectiva de Tempo Futuro (FTPT)}

Tal como sinalizado por Schmitt (2010), o surgimento das teorizações acerca da perspectiva de tempo futuro é muito relacionado aos estudos de Joseph Nuttin (1909-1988). Muitos teóricos, tais como Kurt Lewin desde a década de 1930 (Lens, 1993), já haviam se dedicado à investigação da relação entre o comportamento humano e a personalidade relacionada à dimensão temporal do futuro. No entanto, foi Nuttin quem definiu uma das teorias de maior influência nesse campo de estudos: a FTPT- Future Time Perspective Theory (Teoria da Perspectiva de Tempo Futuro). Trata-se de uma teoria que teve seu auge de desenvolvimento a partir de 1980 e difere das demais por trazer para o campo da motivação uma dimensão de temporalidade, uma vez que focaliza sua investigação nas influências que os objetivos e metas orientadas para o futuro cronológico exercem sobre os comportamentos que se observam no presente (Schmitt, 2010).

Nuttin (1983) dedicou a maior parte de sua vida aos estudos no Laboratório de Psicologia Experimental, em Leuven, na Bélgica. Atuando como docente desde 1946 na Universiteit Leuven, produziu diversas obras, dentre as quais se destaca o livro "Theorie de La motivation Humaine". Tal trabalho é de central importância, pois foi neste texto que o autor se opôs aos modelos anteriores da teoria da motivação, tidos por ele como “instintivos e impessoais". Nuttin então propôs uma teoria cognitiva e relacional da motivação, segundo a qual o caráter contínuo e dinâmico da interação indivíduo-ambiente supõe que as necessidades e motivações sejam personalizadas, uma vez que cada pessoa possui suas representações e seus próprios projetos de vida (Nuttin, 1983). Em síntese, nos principais trabalhos deste pesquisador fica ressaltado que o futuro, em sua dimensão psicológica, é essencialmente e exclusivamente relacionado com a motivação. Retornaremos a este ponto em breve; por hora, delinearemos como se deu a tradição de estudos da Future Time Perspective Theory - FTPT (Teoria da Perspectiva de Tempo Futuro).

Apesar de Nuttin ter contribuído de forma considerável para o desenvolvimento da teoria, foi Willy Lens, seu principal colaborador e atualmente professor da Katholieke Universiteit Leuven, quem efetivamente delineou os principais elementos norteadores deste construto. Para Lens (1993), a perspectiva de tempo futuro se caracteriza pela integração do futuro cronológico no momento presente do indivíduo. Segundo o autor, a perspectiva de tempo futuro é um traço relativamente estável da personalidade, que se desenvolve com base nas características 
pessoais e a partir dela, fixam-se as metas futuras em maiores ou menores espaços (prazos) de tempo.

Partindo desse pressuposto, Lens (1993) especifica três níveis de Perspectiva Futura (PF): extensa, restrita e alongada. Para o autor, jovens que estabelecem metas a serem realizadas num futuro distante são dotados de uma PF extensa e aqueles que fixam objetivos para um futuro próximo possuem uma $P F$ restrita. Há também aqueles que perseguem objetivos que requerem anos de espera e engajamento, estes, segundo Lens, seriam capazes de adiar consideravelmente suas satisfações imediatas e ainda assim, permanecerem voltados para a orientação da meta (Schmitt, 2010).

Na leitura de sua obra, é possível perceber que os indivíduos com PF extensa são considerados como tendo um modelo de planejamento sadio e estável. Isto fica evidente em conclusões como as seguintes (Lens, 1993, p. 80): a) indivíduos dotados de PF extensa percebem as distâncias temporais como mais curtas do que aqueles que estão orientados por uma PF restrita. Os primeiros são mais aptos a suportar gratificações mais tardias quando comparados com os segundos; b) aqueles que possuem PF extensa antecipam melhor as consequências em longo prazo de suas ações no presente, além de atribuírem maior valor aos objetivos distantes e manterem-se mais motivados para perseguir os objetivos; c) a satisfação, a perseverança e o esforço despendido na execução de uma tarefa são maiores nos sujeitos com PF extensa ou alongada. A probabilidade de passar da planificação à ação é mais elevada para esses sujeitos, se os objetivos intermediários servirem como etapas na busca de um objetivo final distante; d) aqueles que são dotados de uma perspectiva futura extensa são mais aptos a transformar seus desejos ou suas vontades em intenções comportamentais e, posteriormente, em ações. Essa passagem da cognição à ação deverá ser facilitada por uma PF que inclua localizações temporais precisas.

Observando cronologicamente, podemos constatar que um dos primeiros trabalhos a relacionar jovens e suas estimativas quanto ao futuro foi realizado no início da década de 1980 na Inglaterra, por De Volder e Lens (1982). Em seu estudo com adolescentes, descobriram que tanto o desempenho com notas como a persistência nos estudos estavam positivamente relacionados com alta valorização de metas para um futuro distante. Esclarecem ainda que a perspectiva do tempo futuro não se reduz a uma simples fantasia quanto ao futuro. Propuseram que nessa perspectiva encontram-se dois aspectos distintos: o dinâmico e o cognitivo - o aspecto dinâmico da FTP consiste na disposição de um indivíduo para atribuir alta importância a metas em um futuro distante. Já o aspecto cognitivo da FTP é formado pela disposição de compreender as consequências em longo prazo do comportamento real, que se reflete no conceito de valor instrumental de um ato comportamental. Esta distinção de aspectos dinâmicos e cognitivos foi posteriormente retomada por Husman e Lens (1999).

Van Calster, Lens e Nuttin (1987), em seu estudo com 230 alunos ingleses com idade entre 17 e 19 anos, concluíram que quando estes percebem a escolarização como importante para o sucesso no futuro são significativamente mais motivados do que aqueles que a percebem como menos importante; considerando então a relação entre o que os jovens esperam do futuro com o modo como se planejam para ele. A atitude dos jovens com relação ao futuro apareceu também como variável crítica no estudo de Lens e Decruyenaere (1991).

Nos últimos anos, tais conclusões ainda vêm recebendo uma expressiva atenção por parte dos pesquisadores e demais estudiosos no campo da educação, a exemplo disso, a Educational Psychology Review publicou em 2004, dois números dedicados, exclusivamente, a essa temática com o título "Effects of time perspective on student motivation". Em um desses números, podemos encontrar o estudo de Simons, Vansteenkiste, Lens e Lacante (2004), realizando, por meio de uma aproximação com a Teoria da Perspectiva de Tempo Futuro, interessantes teorizações sobre a origem motivacional das metas futuras. Outros diversos estudos dedicados ao campo da educação e a investigar a relação entre a perspectiva futura e a construção de metas e ao desempenho acadêmico também foram realizados, encontrando resultados semelhantes: quanto mais extensa a perspectiva de tempo futuro melhor o desempenho em notas e a o engajamento dos estudantes em suas atividades escolares (Carvalho, Pocinho, \& Silva, 2010; Jang \& Liu, 2012; Lee, McInerney, Liem, \& Ortiga, 2010; Peetsma \& Van Der Veen, 2011).

Günther e Günther (1998) foram uns dos pioneiros em trabalhos nacionais realizados a respeito das estimativas de futuro de jovens. Realizaram a pesquisa intitulada: "Brasílias pobres e Brasílias ricas: perspectivas de futuro de adolescentes". Investigando como adolescentes brasilienses percebiam o futuro a partir da estrutura de oportunidades que lhes era disponível, relacionando variáveis socioeconômicas (como idade, gênero, série, trabalho, escola que frequentam) com as perspectivas de futuro apresentadas por eles. O estudo contou com 335 jovens, com idade média de 16 anos e os dados foram coletados em cinco escolas: uma particular, uma pública e três destinadas a jovens carentes e/ou em situação de rua. Os resultados indicaram que adolescentes que frequentavam uma escola privada e cursavam séries mais avançadas, revelaram maiores expectativas de concluir o segundo grau e entrar para a universidade. Os jovens alunos de escolas públicas e com menor renda, por outro lado, em sua maioria, tenderam a não esperar um emprego que garanta boa qualidade de vida, nem esperar possuir casa própria.

Outros estudos brasileiros fundamentados na FTPT foram publicados. Oliveira, Pinto e Souza (2003) investigaram as perspectivas de futuro de adolescentes de diferentes inserções sociais, também na cidade de Brasília. Seus resultados demonstraram que havia, no momento da investigação, níveis semelhantes de preocupação com a carreira universitária e profissional e que eles, independentemente do gênero e do contexto institucional, apresentavam perspectivas mais positivas em relação à continuidade dos estudos que em relação à transição ao mundo do trabalho. Mesmo investigando amostras compostas por jovens de diferentes localidades na cidade de Brasília, na discussão de seus resultados Günther e Günther (1998) e Oliveira et al. (2003) abordam seus grupos de participantes como um todo, pontuando que apesar de suas diferenças sociodemográficas os jovens compartilham sentimentos de incerteza com relação ao futuro e demandam maior orientação educacional voltada ao vestibular e ao ingresso no mercado de trabalho. Em síntese, 
podemos concluir que, ainda que estes estudos tenham tido o objetivo de investigar as diferenças entre participantes de diferentes grupos sociais, terminaram por destacar suas semelhanças enquanto jovens a despeito das dificuldades que alguns deles encontrem em virtude de sua situação social.

Locatelli, Bzuneck e Guimarães publicaram em 2007 o estudo "A motivação de adolescentes em relação com a perspectiva de tempo futuro", preocupados em investigar como a escolarização pode funcionar como promotora de motivação para os jovens, facilitando a construção de seus projetos para o futuro. Os resultados indicaram diferenças quanto ao grau de definição/indefinição dos jovens sobre sua escolha profissional e os autores partiram da FTPT para argumentar a necessidade de programas motivacionais nas escolas e capacitação dos professores como figuras de influência na vida de seus alunos.

Pratta e Santos (2007) investigaram as opiniões de 568 adolescentes e jovens entre 14 e 20 anos de idade, estudantes do ensino médio em 20 escolas públicas e privadas no município de São Carlos-SP, sobre o relacionamento familiar e seus planos para o futuro, comparando grupos de jovens usuários e não usuários de drogas. Os resultados mostraram que aspectos referentes à "dinâmica familiar" são relevantes, tanto do ponto de vista positivo como negativo. No grupo de adolescentes que já utilizaram ou utilizam drogas, o principal tema foi a "falta de diálogo" na família. Os adolescentes não usuários, por sua vez, apresentaram uma porcentagem significativamente maior na categoria "nada", ou seja, não vêem necessidade de melhorar algo no relacionamento com os pais, uma vez que se consideram contentes e satisfeitos com eles. Os principais planos para o futuro apresentados pelos adolescentes de ambos os grupos envolveram questões referentes à família e à preparação e atuação profissional. A situação social desses jovens não foi analisada como variável de influência.

Estes estudos nacionais, apesar de considerarem grupos de diferentes situações sociais (escolas públicas e privadas, moradores de diferentes localidades urbanas e classes sociais distintas), não incluem essa variável em suas análises e discussões de resultados. A leitura dos dados parte dos conceitos motivacionais e cognitivos da FTPT, e o contexto sócio-demográfico fica em segundo plano ou não é nem sequer retomado nas considerações finais dos artigos.

\section{Teoria da Autodeterminação (SDT)}

A Teoria da Autodeterminação (SDT- Self Determination Theory) foi proposta por Edward Deci e Richard Ryan com o objetivo de compreender os componentes da motivação intrínseca e extrínseca e os fatores relacionados com a sua promoção (Deci \& Ryan, 2000). Nessa perspectiva, são abordadas a personalidade e a motivação humana, concentrando-se nas tendências evolutivas, nas necessidades psicológicas inatas e nas condições favoráveis à motivação, ao funcionamento social e ao bem estar pessoal. No contexto da pesquisa educacional, a motivação intrínseca tem sido relacionada ao envolvimento dos alunos com as tarefas de aprendizagem, pela preferência por desafios, persistência, esforço, uso de estratégias de aprendizagem, entre outros resultados positivos (Guimarães \& Boruchovitch, 2004).

Baseados na SDT, Deci e Ryan $(1995,2000)$ relacionaram o grau de utilidade da meta futura (alto vs. baixo) percebido por 137 universitários, estudantes de química, com o tipo de regulação motivacional (interna vs. externa). Concluíram que os indivíduos dotados de percepção de alta utilidade e regulados intrinsecamente são mais propensos à obtenção de resultados positivos na aprendizagem e no rendimento acadêmico.

Em busca de uma taxonomia da motivação humana por meio de da SDT, Vansteenkiste, Soenens, Verstuyf e Lens (2009) investigaram as orientações motivacionais relacionadas à noção de perspectiva futura, realizando um levantamento de estudos com amostras coletadas em ambientes educativos. O estudo identificou a predominância de duas categorias de metas nos resultados das pesquisas com estudantes em geral: as metas futuras intrínsecas (future intrinsic goal) e as metas futuras extrínsecas (future extrinsic goal). Posteriormente, seus resultados em ambientes educativos (Vansteenkiste et al., 2009) e em ambientes esportivos (Vansteenkiste, Matos, Lens, \& Soenens, 2007) apontam que sujeitos que adotam metas futuras intrínsecas possuem maior autonomia, desenvolvem maior senso de instrumentalidade em relação às ações presentes, apresentam resultados mais favoráveis e persistem mais nos comportamentos que se orientam às metas futuras. Assim como a FTPT, o impacto da $S D T$ no contexto educacional também é expressivo. Na busca bibliográfica realizada, grande parte dos artigos encontrados dedica-se a aplicações da Teoria da Autodeterminação à interpretação dos resultados acadêmicos de estudantes e professores (Black \& Deci, 2000; Deci, Koestner, \& Ryan, 2001; Deci, Vallerand, Pelletier, \&, Ryan, 1991; Filak \& Sheldon, 2003; Garn, Matthews, \& Jolly, 2010; Ryan \& Deci, 2000; Ryan \& Niemiec, 2009; Ryan \& Weistein, 2009; Ryan, Stiller, \& Lynch, 1994; Tabachnick, Miller, \& Relyea, 2008; Wang, 2008). É importante destacar, entre estes, o artigo de Bilde, Vanteenkiste e Lens (2011), primeiro trabalho a articular conceitos da FTPT e da SDT em um estudo empírico. Os autores propõem que os processos cognitivos de perspectiva futura e os aspectos motivacionais da autodeterminação estariam, de fato, interligados. Para a amostra de estudantes abordada no estudo, houve uma associação significativa entre as categorias motivacionais (intrínseca e extrínseca) e a perspectivação extensa do futuro, porém a relação entre as teorias não foi retomada por nenhum outro artigo publicado até a revisão realizada neste estudo.

\section{Processos neurais e cognitivos associados ao desen- volvimento de metas para o futuro}

$\mathrm{Na}$ investigação de fatores específicos relacionados ao desenvolvimento de metas futuras, encontramos alguns estudos sobre diversos temas, relacionados a seguir.

Em um estudo teórico, Schacter e Addis (2009) trabalharam com hipóteses semelhantes, reunindo evidências das ciências cognitivas, estudos da memória e neurofisiologia para defender que a capacidade humana de imaginar eventos futuros depende dos mesmos mecanismos cognitivos e neurais utilizados para acessar a memória de eventos passados. Os autores dedicam-se especialmente a demonstrar que a mente processa passado e futuro por meio de processos cerebrais semelhantes.

Natanzon e Ferguson (2012) testaram se o efeito da exposição à visão de movimentos para frente é cognitivamente associado com a motivação para realização de metas e objetivos 
futuros. Expondo os participantes a simples sinais visuais de movimento para frente, ou não, e em seguida medindo a sua motivação para a realização, os pesquisadores encontraram uma relação significativa entre os dois eventos, sugerindo que a ativação de determinadas áreas cerebrais afetam a cognição futura e influi sobre mecanismos motivacionais.

Também em D'Argembeau et al. (2010) encontramos evidências relacionadas ao mapeamento cerebral das metas futuras. Em seu estudo, os pesquisadores constataram que imaginar o futuro ativa especialmente a região ventral medial do córtex pré-frontal e córtex posterior cingulado. Estas ativações cerebrais foram sobrepostas em uma segunda tarefa, na qual os participantes respondiam a avaliações semânticas de autoconhecimento (ou seja, fazer julgamentos sobre seus próprios traços de personalidade), sugerindo que estas regiões cerebrais abarcam uma gama de processos que avaliam, codificam e contextualizam a relevância pessoal das metas futuras.

\section{Discussão e a contribuição da Psicologia Evolucionista}

Em 1963, Niko Tinbergen, um dos principais fundadores da Etologia, a primeira disciplina a formalizar o estudo do comportamento sob a perspectiva da teoria evolucionista darwiniana, mostrou que para investigarmos um atributo ou comportamento precisamos responder a quatro "questões". São elas: 1) Qual a história evolutiva daquele comportamento? Qual a causa filogenética do comportamento? 2) Por que aquele comportamento confere maior habilidade de sobrevivência e reprodução aos indivíduos que o apresentam? Qual sua função ou causa final (ultimate cause)? 3) Quais foram os fatores que modelaram o desenvolvimento do comportamento ao longo da vida daqueles indivíduos? Como se dá a ontogênese daquele comportamento? 4) Por que o indivíduo comportou-se daquela maneira naquele momento particular? Qual a causa imediata ou próxima?

Observa-se que dentre os estudos revistos até aqui, alguns conceitos centrais podem ser destacados. O modelo abaixo (Figura 1) apresenta uma tentativa de síntese dos principais construtos e processos envolvidos na construção de metas e estimativas futuras conforme a revisão realizada por este trabalho.

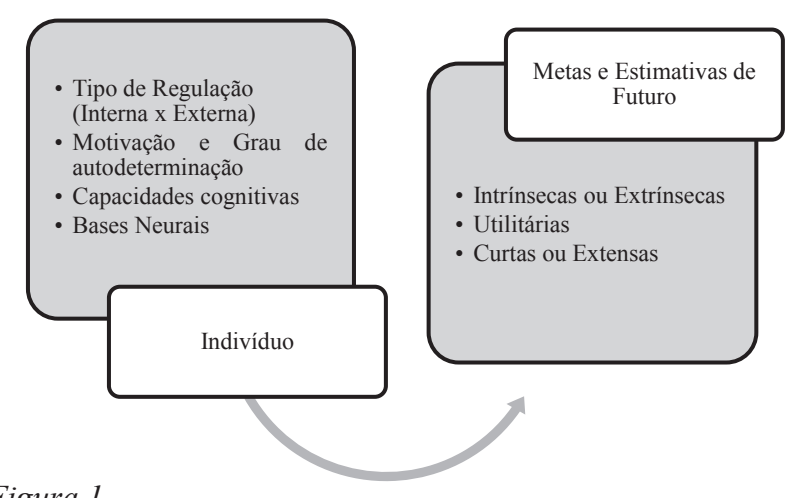

Figura 1

Modelo clássico do estudo das estimativas e metas para o futuro
Considerando desde as primeiras contribuições de Nuttin (1983), passando pela sistematização da teoria realizada por Lens (1993) e revisando investigações de outros pesquisadores, surgem algumas questões importantes: A motivação, personalidade e tomada de decisão, assim como outros processos subjacentes, são os únicos fatores a serem considerados quando estudamos as orientações para o futuro? Pensando nas questões propostas por Tinbergen (1963), as causas últimas, funcionais, filogenéticas e ontogenéticas estão bem explicadas pelo modelo cognitivo/ motivacional oferecido pela FTPT e pela STD?

Buscando responder a estas questões, apresentar-se-á a teoria do Desconto do Futuro, uma proposta evolucionista para o estudo do tema.

A habilidade humana de considerar o tempo e a capacidade de fazer sacrifícios no presente visando obter algo melhor no futuro são parte significativa do sucesso da espécie (Suddendorf \& Corballis, 1997). A evolução da mente humana permitiu-nos a possibilidade de viajar no tempo por meio de processos cerebrais específicos tais como os descritos nos estudos acima mencionados (i.e. D'Argembeau et al., 2010), projetando-nos psicologicamente em situações futuras para medir o valor das recompensas e motivarnos a adiá-las para um tempo mais distante.

A Psicologia Evolucionista busca explicar o funcionamento de nossa mente adaptada, produto da seleção natural, no ambiente de adaptação evolucionária (Barkow, Cosmides, \& Tooby, 1992). A abordagem evolucionista da psicologia, em seu modo diferenciado de explicação dos fenômenos humanos, nos apresenta alguns pressupostos para a compreensão dos comportamentos humanos frente ao futuro, em especial para os sujeitos jovens. Planejar e investir no futuro são condutas relacionadas a uma série de fatores, e tem um histórico ancestral a ser considerado. Respondendo às duas primeiras das quatro questões propostas por Niko Tinbergen (1963), (descritas no tópico anterior) os psicólogos evolucionistas canadenses Martin Daly e Margo Wilson nos explicam as causas filogenéticas e as causas funcionais deste comportamento. Apropriandose desse termo, oriundo da economia, Daly e Wilson (2005) desenvolveram o conceito de Desconto do Futuro, uma tendência comum a todos os organismos vivos (humanos ou não) em preferir o presente em detrimento do futuro em suas decisões, condutas e planejamentos. Esta tendência traduz-se em uma adaptação de nossa mente ancestral que se firmou como estratégia evolutiva, pois, segundo os autores, algum grau de preferência pelo aqui e agora é geralmente adaptativo por no mínimo duas razões: garantir recursos para reproduzir-se o mais cedo possível garante mais sucesso do que adiar essa decisão e; quanto mais distante o prazo para a aquisição de um certo bem ou recompensa, maior é a probabilidade que estes possam ser perdidos (Daly \& Wilson, 2005).

Sendo assim, descontar o futuro é uma estratégia de nossa espécie que, assim como as outras, possui caráter adaptativo (funcional). Essa adaptação, ou funcionalidade, do desconto do futuro apresenta algumas variações. Os homens, por exemplo, tendem a descontar mais o futuro do que mulheres e, isto se relaciona com três aspectos de nossa história: em primeiro lugar, a espécie humana se desenvolveu em condições nas quais o investimento parental materno era obrigatório e o paterno não. 
Dessa forma, a probabilidade de sucesso reprodutivo masculino estava diretamente relacionada à quantidade de recursos que o macho possuía. Em segundo lugar, a probabilidade de morrer sem deixar descendentes é muito maior para homens do que para mulheres. Isto se dá porque, em terceiro lugar, a taxa de mortalidade entre os homens também é maior, o que resulta numa estratégia de vida e em comportamentos mais arriscados, mais agressivos e mais impulsivos que os das mulheres (Daly \& Wilson, 2005).

Com isso, vemos que há um histórico de causas filogenéticas e funcionais relacionadas ao comportamento humano frente ao futuro e que nossa mente integra tais estratégias herdadas da evolução a outros elementos e processos para chegar ao resultado final e definir como planejaremos nosso futuro. Quais seriam as taxas ou proporções ideais para se descontar o futuro? A resposta para tal questão acompanha as duas outras causas levantadas por Tinbergen (1963) e listadas no fim do tópico anterior: as causas próximas e as ontogenéticas. George Williams (1957, citado por Daly \& Wilson, 2005), responde a essa questão, atribuindo ao contexto a tarefa de modelar o desconto do futuro. Segundo este autor, as taxas ótimas para esse comportamento tendem a covariar em virtude do índice de mortalidade no local. A pressão evidente do risco e as ameaças à sobrevivência funcionariam, nesses casos, como causas próximas.

Assim como George Williams, pesquisadores da Universidade de Detroit também consideram o risco do contexto como uma variável mediadora para o desconto e expectativas de futuro, porém, acrescentam ao modelo a interação com outra variável próxima: a estabilidade da família e do cuidado parental recebido na infância (Hill, Jenkins, \& Farmer, 2008). Segundo esses autores, há uma relação entre as medidas de imprevisibilidade familiar na infância e os comportamentos de risco, e uma segunda relação entre esta última variável e as estimativas e desconto do futuro. As características da família agem, indiretamente, como causas próximas para o desconto e expectativas de futuro e também para as expectativas de vida.

No estudo das causas ontogenéticas, Green, Myerson e Ostaszewski (1999) demonstraram que as taxas de desconto do futuro declinam ao longo do ciclo de vida humano, são muito maiores em crianças e adolescentes do que em indivíduos de setenta anos, por exemplo. Kirby e Marakovic (1996) também comprovaram que a ontogênese do desconto do futuro segue este mesmo padrão. Segundo esses autores, esse processo tem seu ápice durante os primeiros anos da puberdade, em decorrência do desenvolvimento do cérebro, mais especificamente das estruturas pré-frontais e subcorticais (Ersner-Hershfield, Wimmer, \& Knutson, 2009). A tendência em privilegiar o presente é intensa em crianças e adolescentes e essa característica ontogenética do desconto do futuro guarda relação não apenas com a idade do individuo, mas com as transformações cerebrais e hormonais da puberdade e com a impulsividade característica do comportamento adolescente.

Hershfield, Garton, Ballard, Samanez-Larkin e Knutson (2009) demonstraram que o desenvolvimento da noção de self está relacionado com a avaliação do futuro e também com a capacidade de projetar uma imagem de si ao longo do tempo (Hershfield et al., 2009). Sobre esta ligação entre ontogênese da noção de self e do desconto do futuro, alguns teóricos têm argumentado que o desconto intertemporal emerge do conflito de interesses entre diferentes "selves temporais" (Parfit, 1971, 1987; Schelling, 1984 citados por Hershfield et al., 2009). De acordo com essa visão, para uma pessoa, a força da ligação entre seu self presente seu self futuro tende a declinar na medida em que aumenta a distância de tempo entre eles. Assim, preservar ganhos para um self muito distante é o mesmo que abrir mão de seus bens atuais e doá-los a um estranho. As metas que desenvolvemos para o futuro falam, na verdade, de nossas necessidades atuais transferidas para um projeto adiado, mas que guarda íntima relação com o self atual e suas características. Segundo Hershfield et al., (2009), essa perspectiva de "múltiplos selves" tem implicações mais focadas no planejamento financeiro. Se as pessoas tendem a considerar seu self futuro como um estranho, racionalmente (e evolutivamente) não há razões para privar-se de ganhos atuais e reservá-los para um estranho. Esta relação também sofre influência da idade, pois segue a ontogênese da noção de self.

Nesta interação entre desenvolvimento e a noção de futuro, encontramos em Del Giudice e Belsky (2011) algumas evidências importantes. Primeiramente, o desenvolvimento é concebido como um processo de interação de fatores genéticos e ambientais. Em segundo lugar, as interações próximas (com a família, com os pares, etc.) modelam este processo de interação, possibilitando ou suprimindo o desenvolvimento de uma habilidade/característica em detrimento de outra (ex: autonomia x interdependência). Por fim, os autores listam uma série de variáveis contextuais e subjetivas que compõem a equação do comportamento humano frente ao futuro. Sendo assim, não importam apenas os fatores intrínsecos, cognitivos/motivacionais para a construção de um projeto futuro, tal como defendido pela Teoria da Autodeterminação e pela Teoria da Perspectiva de Tempo Futuro e também demonstrado no esquema anterior (Figura 1). As extrínsecas também são fundamentais.

Segundo Geary e Bjorklund (2000), o processo epigenético gera tanto características fenotípicas comuns aos membros da espécie humana como variações no comportamento adaptadas às ecologias locais. Com uma proposta semelhante, Del Giudice e Belsky (2011) afirmam que o desenvolvimento humano pode ser bem explicado em termos de estratégias de história de vida (Life History Theory), e tais estratégias para o sujeito consistem, essencialmente, em identificar as soluções melhor adaptadas para os vários desafios presentes em seu contexto. As variações no ambiente socioecológico (riscos e mortalidade, principalmente) dão as pistas necessárias para que possamos responder estrategicamente a estes desafios, acessando os mecanismos geneticamente evoluídos de nossa mente para estabelecer metas futuras adaptadas a condições especiais de desenvolvimento. Tais condições de desenvolvimento, por fim, possibilitam ao sujeito a adoção de dois possíveis estilos de estratégias para a organização de seu esforço vital: uma estratégia rápida (fast $\mathrm{LH}$ ) ou uma estratégia alongada (slow $\mathrm{LH}$ ).

De acordo com os autores acima, nossa mente interage com variáveis específicas do contexto, direcionando o comportamento a um desses tipos de estratégias. Quando a morbidade-mortalidade é alta em um dado contexto (devido à carga de patógenos, por exemplo), é adaptativo favorecer a reprodução mais precocemente, dirigindo o projeto de vida para prazos curtos e metas mais voltadas para a quantidade de experiências e sensações, mesmo que isso implique em risco para o sujeito. O risco de mortalidade extrínseca, nesse caso, consiste em uma taxa de mortalidade local que não pode ser prevenida alterando-se o comportamento do sujeito, e é 
um dos fatores cruciais para a definição de qual estilo de estratégia será adotado pelo indivíduo em seu ciclo vital.

A imprevisibilidade do contexto, a escassez de recursos e a competição extrema entre os indivíduos favorecem, então, a adoção de uma estratégia de vida rápida, enquanto um cenário de maior estabilidade, recursos suficientes e proteção favorecem o desenvolvimento de uma estratégia de vida alongada. É justamente a variação desses indicadores externos que altera os custos e benefícios envolvidos nas estratégias de vida. Em síntese, as estratégias de LH desempenham um papel importante na organização do comportamento. Podemos identificar um conjunto de traços relacionados que se espera que covariem ao longo da história de vida: escolha de parceiros e estratégias sexuais, busca por status, a agressividade, cooperação, altruísmo, o comportamento de risco, o apego romântico, os estilos parentais e, principalmente, a perspectiva do tempo futuro. Correlações dentro deste conjunto de comportamentos e variáveis têm sido documentadas também em animais não humanos (Dingemanse \& Reale, 2005) e em seres humanos (Figueredo et al., 2006).

Del Giudice e Belsky (2011) apresentam um panorama diferenciado para o estudo do desenvolvimento no ciclo vital. A partir dele podemos compreender que variáveis devem receber maior atenção na análise das metas e projetos futuros dos jovens. Se relembrarmos as teorias clássicas no estudo do tema, nota-se que o panorama evolucionista, proposto pelos autores, amplia não apenas o número de variáveis envolvidas no processo, como também modifica o modo de análise dessas variáveis, indicando que demandas específicas do contexto em interação com características do indivíduo estão no centro da elaboração das metas e expectativas futuras. Com base nesses argumentos, podemos pensar em um segundo modelo para o estudo do tema:
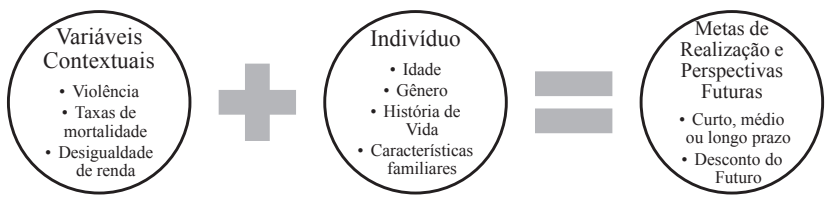

Figura 2

Proposta de modelo para o estudo das metas futuras com base na abordagem evolucionista

A abordagem evolucionista coloca indivíduo e contexto como entes em interação para compreendermos o complexo fenômeno das perspectivas e expectativas de futuro, porém, este não é o único fator diferencial desta abordagem, mas também a forma como individuo e contexto são compreendidos. A Psicologia Evolucionista não apenas aponta indivíduo e contexto como elementos do processo como também destaca quais variáveis específicas devem ser observadas em ambos, além de teorizar acerca das interações esperadas entre tais variáveis. Conforme demonstrado no esquema acima (Figura 2).

\section{Considerações finais}

A revisão realizada por este artigo permitiu identificar a predominância de três eixos teóricos na produção científica sobre metas de realização e objetivos futuros de jovens. Os estudos baseados na Teoria da Perspectiva de Tempo Futuro, proposta por Joseph Nuttin (1983) e Willy Lens (1993), privilegiam os aspectos de temporalidade da motivação humana em relação ao futuro e os processos cognitivos envolvidos no processo de planejamento e perspectivas distais.

Concentrados em sua maioria no campo da educação, os estudos baseados na Teoria da Autodeterminação (Deci \& Ryan, 1995) buscam investigar os componentes intrínsecos e extrínsecos da motivação futura, suas necessidades inatas e condições favoráveis/desfavoráveis ao bem estar pessoal.

Por fim, observou-se que os estudos de neuroimagem e cognição identificaram processos cerebrais ligados às metas futuras e apontam que há ativação de regiões semelhantes àquelas envolvidas no autorrelato e no ato da exposição visual a movimentos em direções específicas.

No entanto, nota-se que os três eixos teóricos encontrados não oferecem um modelo integrativo para o estudo das metas de realização e objetivos futuros, assim como não consideram as quatro causas propostas por Tinbergen (1963) para o estudo de fenômenos comportamentais. Além disso, os fatores contextuais e sociodemográficos ligados ao desenvolvimento humano, em especial ao desenvolvimento durante a juventude, não são levados em conta.

Este artigo, por meio de da Teoria do Desconto do Futuro (Daly \& Wilson, 2005) e da Teoria das Estratégias de História de Vida (Del Giudice \& Belsky, 2011), propôs a perspectiva evolucionista da psicologia para o estudo do tema em questão.

A inovação trazida pela perspectiva evolucionista é a de colocar dentro do escopo da Psicologia o estudo das causas últimas, evolutivas, contrariando a tradição histórica da área em estudar apenas as causas próximas. Não por acaso, a Psicologia Evolucionista é frequentemente definida como uma abordagem à Psicologia e não como uma área específica, como Psicologia da Personalidade ou do Desenvolvimento, por exemplo. Nesse sentido, ela é proposta como uma forma de pensar a Psicologia (evolutivamente) que pode ser aplicada a qualquer tema dentro dela (Gaulin \& McBurney, 2001).

A partir desse arcabouço teórico, este trabalho propõe o estudo das perspectivas futuras dos jovens a partir do modelo evolucionista, considerando não apenas variáveis intrínsecas, mas a interação e o valor adaptativo das estratégias adotadas pelos sujeitos. Capaz não apenas de integrar os componentes intrínsecos e extrínsecos da construção de metas futuras, a Psicologia Evolucionista também inclui o estudo dos dados contextuais e sociodemográficos ligados ao risco e a mortalidade como importantes para abordar esse tema.

Esta proposta tem implicações para a compreensão do comportamento de jovens e para o desenvolvimento de programas de intervenção visando à promoção de saúde nesta faixa etária.

\section{Referências}

Barkow, J. H., Cosmides, L., \& Tooby, J. (Orgs.). (1992). The adapted mind. Oxford: Oxford University Press.

Baron, J. (2008). Thinking and deciding. Cambridge: Cambridge University press. 
Baumeister, R. F., \& Leary, M. R. (1995). The need to belong: Desire for interpersonal attachments as a fundamental human motivation. Psychological Bulletin, 117, 497-529.

Bilde, J., Vansteenkiste, M., \& Lens, W. (2011). Understanding the association between future time perspective and self-regulated learning through the lens of self-determination theory. Learning and Instruction, 21(3), 332-344.

Black, A. E., \& Deci, E. L. (2000). The effects of instructors' autonomy support and students' autonomous motivation on learning organic chemistry: A selfdetermination theory perspective. Science Education, 84, 740-756.

Carvalho, R. G., Pocinho, M., \& Silva, C. (2010). Comportamento adaptativo e perspectivação do futuro: Algumas evidências nos contextos da educação e da saúde. Psicologia: Reflexão e Crítica, 23(3), 554-561.

Daly, M., \& Wilson, M. (2005). Carpe diem: Adaptation and devaluing the future. The Quarterly review of Biology, 80(1), 55-61.

Damon, W., Menon, J., \& Bronk, K. C. (2003). The development of purpose during adolescence. Applied Developmental Science, 7(3), 113-128.

D’Argembeau, A., Stawarczyk, D., Majerus, S., Collete, F., Van Der Linden, M., Feyers, ... Salmon, E. (2010). The neural basis of personal goal processing when envisioning future events. Journal of Cognitive Neuroscience, 22(8), 1701-1713. doi: 10.1162/jocn.2009.21314

Deci, E., \& Ryan, R. (1995). Intrinsic motivation and self determination in human behavior. Nova Iorque: Plenum Publishing Co.

Deci, E., \& Ryan, R. (2000). The "what" and "why" of goal pursuits: Human needs and the self-determination of behavior. Psychological Inquiry, 11(4), 227-268.

Deci, E. L., Koestner, R., \& Ryan, R. M. (2001). Extrinsic rewards and intrinsic motivation in education: Reconsidered once again. Review of Educational Research, 71, 1-27.

Deci, E. L., Vallerand, R. J., Pelletier, L. G., \& Ryan, R. M. (1991). Motivation and education: The self-determination perspective. The Educational Psychologist, 26, 325-346.

Del Giudice, M., \& Belsky, J. (2011). The development of life history strategies: Toward a multi-stage theory. In D. M. Buss \& P. H. Hawley (Orgs.), The evolution of personality and individual differences. (pp. 154-176). Nova Iorque: Oxford University Press.

De Volder, M. L., \& Lens, W. (1982) Academic achievement and future time perspective as a cognitive-motivational concept. Journal of Personality and Social Psychology, 42(3), 566-571.

Dingemanse, N. J. , \& Réale, D. (2005). Natural selection and animal personality. Behaviour, $142,1165-1190$.

Erikson, E. H. (1968). Identity: youth and crisis. Nova Iorque: Norton.

Ersner $\square$ Hershfield, H., Wimmer, G. E., \& Knutson, B. (2009). Neural evidence for self $\square$ continuity in temporal discounting. Social Cognitive and Affective

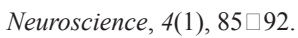

Figueredo, A. J., Vásquez, G., Brumbach, B., Schneider, S. M. R., Sefcek, J. A., Tal, I. R., \& Jacobs, W. J. (2006). Consilience and life history theory: From genes to brain to reproductive strategy. Developmental Review, 26, 243-275.

Filak, V., \& Sheldon, K. M. (2003). Student psychological need satisfaction and college teacher-course evaluations. Educational Psychology, 23, 235-247.

Garn, A. C., Matthews, M. S., \& Jolly, J. L. (2010). Parental influences on the academic motivation of gifted students: A self-determination theory perspective. Gifted Child Quarterly, 54, 263-272.

Gaulin, S. J. C., \& McBurney, D. H. (2001). Psychology: An evolutionary approach. Upper Saddle River: Prentice-Hall.

Geary, D. C., \& Bjorklund, D. F. (2000). Evolutionary developmental Psychology. Child Development, 71(1), 57-65.

Green, L., Myerson, J., \& Ostaszewski, P. (1999). Discounting of delayed rewards across the life span: age differences in individual discounting functions. Behavioral Processes, 46(1), 89-96.

Guimarães, S. E. R., \& Boruchovitch, E. (2004). O estilo motivacional do professor e a motivação intrínseca dos estudantes: Uma perspectiva da teoria da autodeterminação. Psicologia: Reflexão e Crítica, 17(2), 143-150.

Günther, I. A., \& Günther, H. (1998). Brasílias pobres, Brasílias ricas: Perspectivas de futuro entre adolescentes. Psicologia Reflexão e Crítica, 11(2), 191-207. Hershfield, H. E., Garton, M., Ballard, K., Samanez-Larkin, G., \& Knutson, B. (2009). Don't stop thinking about tomorrow: Individual differences in future self-continuity account for saving. Judgment and Decision Making, 4(4), 280-286.

Hill, E., Jenkins, J., \& Farmer, L. (2008). Family unpredictability, future discounting, and risk taking. The Journal of Socio-Economics, 37, 1381-1396.

Husman, J., \& Lens, W. (1999). The role of the future in student motivation. Educational Psychologist, 34, 113-125.

Jang, L. Y., \& Liu, W. C. (2012). Achievement goals and achievement emotions: A cluster analysis of students' motivation. European Journal of Psychology of Education, 27, 59-76.

Kirby, K. N., \& Marakovic, N. N. (1996). Delay-discounting probabilistic rewards: Rates decrease as amounts increase. Psychonomic Bulletin and Review, 3(1), 100-104.

Lee, J., McInerney, D. M., Liem, G. A. D., \& Ortiga, Y. P. (2010). The relationship between future goals and achievement goal orientations: An intrinsicextrinsic motivation perspective. Contemporary Educational Psychology, 35(4), 264-279.

Lens, W. (1993). La signification motivationnelle de la perspective future. Revue québécoise de psychologie, 14(1), 69-83.

Lens, W., \& DeCruyenaere, M. (1991). Motivation and de-motivation in secondary education: Student characteristics. Learning and Instruction, 1(2), 145-159.

Locatelli, A. C. D., Bzuneck, J. A., \& Guimarães, S. E. R. (2007). A motivação de adolescentes em relação com a perspectiva de tempo futuro. Psicologia: Reflexão e Crítica, 20, 268-276.

Loevinger, J. (1976). Ego development. San Francisco: Jossey-Bass, Inc.

Marcia, J. E. (2002). Identity and psychosocial development in adulthood. Identity, 2, 7-2.

Montemayor, R. (1983). Parents and adolescents in conflict: All families some of the time and some families most of the time. Journal of Early Adolescence, 3, 83-103.

Natanzon, M., \& Ferguson, M. J. (2012). Goal pursuit is grounded: The link between forward movement and achievement. Journal of Experimental Social Psychology, 48(1), 379-382.

Nuttin, J. (1983). Teoria da motivação humana. São Paulo : Loyola.

Oliveira, M. C. S. L., Pinto, R. G., \& Souza, A. S. (2003). Perspectivas de futuros entre adolescentes: Universidade, trabalho e relacionamentos na transição para a vida adulta. Temas em Psicologia, 11, 16-27.

Peetsma, T., \& Van Der Veen, I. (2011). Relations between the development of future time perspective in three life domains, investment in learning, and academic achievement. Learning and Instruction, 21, 481-494.

Pratta, E. M. M., \& Santos, M. A. (2007). Opiniões dos adolescentes do ensino médio sobre relacionamento familiar e seus planos para o futuro. Paidéia (Ribeirão Preto), 17, 103-114.

Ramos, D. O. (2009). Expectativas de vida e metas para ofuturo de jovens em dois contextos distintos: Uma abordagem evolucionista (Monografia de conclusão do curso de Psicologia). Universidade Estadual do Rio de Janeiro, RJ.

Ryan, R. M., \& Deci, E. L. (2000). Intrinsic and extrinsic motivations: Classic definitions and new directions. Contemporary Educational Psychology, 25, 54-67.

Ryan, R. M., \& Niemiec, C. P. (2009). Self-determination theory in schools of education: Can an empirically supported framework also be critical and liberating? Theory and Research in Education, 7, 263-272.

Ryan, R. M., \& Weinstein, N. (2009). Undermining quality teaching and learning: A self-determination theory perspective on high-stakes testing. Theory and Research in Education, 7, 224-233.

Ryan, R. M., Stiller, J., \& Lynch, J. H. (1994). Representations of relationships to teachers, parents, and friends as predictors of academic motivation and self-esteem. Journal of Early Adolescence, 14, 226-249.

Schacter, D. L., \& Addis, D. R. (2009). Remembering the past to imagine the future 
: A cognitive neuroscience perspective. Militar Psychology, 21, 108-122.

Schmitt, R. (2010). Teoria da perspectiva de tempo futuro: Aplicações preliminares e reflexões voltadas à pesquisa no ensino superior. Revista Educação por Escrito, (1), 5-16.

Simons, J., Vansteenkiste, M., Lens, W., \& Lacante, M. (2004). Placing motivational and future time perspective theory in a temporal perspective. Educational Psychology Review, 16(2), 121-139.

Suddendorf, T., \& Corballis, M. C. (1997). Mental time travel and the evolution of the human mind. Genetic, social, and general psychology monographs, 123, 133-167.

Tabachnick, S. E., Miller, R. B., \& Relyea, G. E. (2008). The relationships among students' future-oriented goals and subgoals, perceived task instrumentality, and task-oriented self-regulation strategies in an academic environment. Journal of Educational Psychology, 100(3), 629-642.

Tinbergen, N. (1963). On aims and methods of ethology. Zeitschrift für
Tierpsychol, 20, 410-433.

Van Calster, K., Lens, W., \& Nuttin, J. (1987). Affective attitude toward the personal future: Impact on motivation in high school boys. American Journal of Psychology, 100, 1-13.

Vansteenkiste, M., Matos, L., Lens, W., \& Soenens, B. (2007). Understanding the impact of intrinsic versus extrinsic goal framing on exercise performance: The conflicting role of task and ego involvement. Psychology of Sport and Exercise, 8(5), 771-194.

Vansteenkiste, M., Soenens, B., Verstuyf, J., \& Lens, W. (2009). What is the usefulness of your schoolwork? The differential effects of intrinsic and extrinsic goal framing on optimal learning. Theory and Research in Education, 7(2), 155-164.

Wang, F. (2008). Motivation and English achievement: An exploratory and confirmatory factor analysis of a new measure for Chinese students of English learning. North American Journal of Psychology, 10, 633-646.

Dandara de Oliveira Ramos, mestre em Psicologia Social pelo Programa de Pós Graduação em Psicologia Social da Universidade do Estado do Rio de Janeiro (PPGPS/UERJ), é doutoranda em Epidemiologia do Programa de Pós-graduação em Saúde Coletiva do Instituto de Medicina Social da Universidade do Estado do Rio de Janeiro (IMS/UERJ). Endereço para correspondência: Rua Moacir de Almeida, 219. Bloco 01, Ap 806. Tomas Coelho- RJ, CEP: 20750-340. Tel: (21)79081296. E-mail: dandararamos2@gmail.com Maria Lucia Seidl-de-Moura, doutora em Psicologia Cognitiva pela Fundação Getúlio Vargas - RJ (1987), livre-docência pela Universidade Federal do Rio de Janeiro (1992) e pós-doutorado em Psicologia Evolucionista na Universidade de São Paulo e na PUC / RJ , é professora titular do Instituto de Psicologia da Universidade do Estado do Rio de Janeiro. E-mail: mlseidl@gmail.com Luciana Fontes Pessôa, doutora e pós-doutora em Psicologia Social pelo Programa de Pós Graduação em Psicologia Social da Universidade do Estado do Rio de Janeiro (PPGPS/UERJ), é professora assistente do departamento de Psicologia da Pontifícia Universidade Católica do Rio de Janeiro (PUC/RJ). E-mail: pessoalf@gmail.com 